\title{
Thyroid Metastasis as the First Manifestation of Renal Clear Cell Cancer
}

\author{
Arturs Ozolins*, Arnis Abolins**, Ilze Strumfa**, Peteris Vaganovs*, Zenons Narbuts*, Janis Gardovskis* \\ * Department of General Surgery, Pauls Stradins Clinical University Hospital, Riga, Latvia \\ **Department of Pathology, Riga Stradins University, Latvia
}

\section{Summary}

Thyroid metastases from extra-thyroid primary tumours are reported to be rare, mostly originating from lung, breast or renal cancer. Renal clear cell cancer (RCCC) is a tumour with great metastatic potential, but solitary metastases, especially in the thyroid gland, are uncommon. Here we report an unusual clinical case. In our patient, metastatic thyroid lesion was diagnosed before the primary RCCC. Surgical treatment yielded cancer-free survival, at present lasting already for 1.5 years.

Key words: Thyroid metastasis, renal clear cell cancer.

\section{AIM OF THE DEMONSTRATION}

Renal cancer represents around 3\% of adult malignancies (7). RCCC is the most frequent histological type. Although the clinical course of RCCC is unpredictable, there is a high probability of metastasis in different locations. Based on recent literature, the most common extra-thyroid malignancies that metastasize to the thyroid gland are renal cell $(48.1 \%)$, colorectal $(10.4 \%)$, lung $(8.3 \%)$, and breast carcinoma $(7.8 \%)$, as well as sarcoma $(4.0 \%)$. Metastases to the thyroid are more common in women than men (female to male ratio $=1.4$ to 1 ) and in nodular thyroid glands $(44.2 \%)$ as described by Chung et al. (2). Here, we aim to discuss a rare case when RCCC metastasis in thyroid is detected before diagnosing primary tumour of the kidney.

\section{CASE REPORT}

A 75-year-old woman was referred from endocrinologists to surgeon due to nodular toxic goiter. She complained about enlarged thyroid gland and periodical shortness of breath during the previous two years. No other complaints were mentioned. Due to hormonally active goiter, daily Thyrozol $5 \mathrm{mg}$ has been prescribed for one year. Preoperative ultrasound examination (US) showed the presence of a multinodular goiter. Scintiscan of the thyroid yielded one "cold" and one "hot" nodule, located in the left and right lobe, respectively. Preoperative fineneedle aspiration biopsy (FNAB) was not accomplished. Total thyroidectomy was performed. The operative findings showed multinodular goiter with dominant elastic soft mass measuring $3 \mathrm{~cm}$ in diameter in left lobe (Figure 1A). There was no apparent extra-thyroidal invasion or lymph node involvement. Pathological grossing revealed soft, grey nodule in left lobe on the background of nodular goiter. Microscopically the nodule was composed of atypical clear cells partially enclosed within connective tissue pseudocapsule (Figure 1B). The atypical cells were large, with optically clear cytoplasm and medium size nuclei. Haemorrhages were observed in stroma, rich in prominent sinusoid-like vessels (Figure $1 \mathrm{C}$ ). To reach evidence-based diagnosis, conventional morphology was supplemented with immunohistochemistry (IHC). By IHC, cells intensively expressed vimentin and epithelial membrane antigen, but lacked CD117, HBME-1, cytokeratin 7, thyroid transcription factor, chromogranin A and calretinin. According to morphological and IHC results, RCCC metastasis in thyroid gland was diagnosed. After the morphological verification of diagnosis, patient was scheduled for computed tomography scan (CT) of the abdomen. Tumour mass measuring $8 \times 9 \times 6 \mathrm{~cm}$ in the posterior part of the left kidney as well as enlarged paraaortic and retroperitoneal lymph nodes were found (Figure 2). Patient underwent laparoscopic left side nephrectomy which was done in standard technique without any complications. Final morphology was RCCC, Fuhrman grade II. Angiogenesis-targeting therapy was started. Control bone scintiscan revealed no metastasis in the bones (Figure 3). Patient has been doing well without any evidence of recurrence for 1.5 years after the kidney operation.

\section{DISCUSSION}

Metastases from extra-thyroid malignancies to the thyroid gland have been reported in $1.4 \%-3 \%$ of all patients who have surgery for thyroid malignancy, whereas the autopsy studies report a wide range of prevalence, from $1.9 \%$ to $24 \%$, for the metastases to the thyroid $(5,7)$. These cancer metastases to the thyroid only rarely are clinically recognized. As for the clinical cases in the literature, the primary tumour is renal cell carcinoma in more than half of the cases. Chen et al. (1) also reported ten patients with thyroid metastases, which had been an isolated manifestation during an 8 -year period, and $50 \%$ of these patients had metastases of renal cell carcinoma to the thyroid.

In clinical practice, a correct diagnosis of metastasis to the thyroid is difficult, since there are no specific clinical features and only the few findings on US or CT investigations are characteristic of secondary thyroid 
lesion (5). Several studies have indicated that abnormal glands (nodular goiter, adenomas, well-differentiated carcinomas, thyroiditis, etc.) are more likely to harbour metastatic disease than normal glands, presumably due to abnormal blood supply resulting in decreased oxygen and iodine content $(3,7)$.

We described the case of a solitary metastasis in the thyroid from RCCC. Thyroid metastasis from RCCC occasionally is found years after primary nephrectomy, but this is unusual case as the metastasis was found before the identification of primary tumour. A detailed clinical history is relevant for the diagnosis of solitary thyroid metastases from RCCC because of the nonspecificity of supplemental tests. FNAB of thyroid masses could be useful in diagnosis of thyroid metastases; this, however, requires information about the extra-thyroid malignancies, so that proper antibodies can be used for IHC studies. Preoperative diagnosis can be improved using IHC on FNAB samples from patients with a history of RCCC and thyroid nodules with an aggressive behaviour (4).

It is important to keep in mind that the thyroid gland can be a site of metastases for a variety of tumours when evaluating a thyroid nodule, especially in a patient with a prior history of malignancy (6). In patients with thyroid lesions and a history of malignant disease, regardless of time elapsed since the initial diagnosis of the primary neoplasm, disease recurrence or progression of malignancy must be considered until proven otherwise (2). Surgical treatment in combination with angiogenesis-targeting therapy has the highest survival rate in case of RCCC.

\section{Conflict of interest: None}

Acknowledgements: The research was carried out within the frames of scientific project RSU ZP 08/2013, supported by Rīga Stradinš̌ university

\section{REFERENCES}

1. Chen H, Nicol TL, Udelsman R. Clinically significant, isolated metastatic disease to the thyroid gland // World J Surg, 1999; 23(2):177 - 180

2. Chung AY, Tran TB, Brumund KT, Weisman RA, Bouvet M. Metastases to the thyroid: a review of the literature from the last decade // Thyroid, 2012; 22(3): $258-268$

3. Heffess CS, Wenig BM, Thompson LD. Metastatic renal cell carcinoma to the thyroid gland: a clinicopathologic study of 36 cases // Cancer, 2002; 95(9): $1869-1878$

4. Narine N, Thiryayi SA, Perera DM. Fine-needle aspiration cytology of renal clear cell carcinoma metastatic to the thyroid gland // Diagn Cytopathol, 2013; 41(9):843 - 845

5. Seki H, Ueda T, Shibata Y, Sato Y, Yagihashi N. Solitary thyroid metastasis of renal clear cell carcinoma: report of a case // Surg Today, 2001; $31(3): 225-229$
6. Sindoni A, Rizzo M, Tuccari G, Ieni A, Barresi V, Calbo L, Cucinotta E, Mallamace A, Trimarchi F, Benvenga S. Thyroid metastases from clear cell renal carcinoma 18 years after nephrectomy // Annales d'endocrinologie, 2010; $71(2): 127$ - 130

7. Zamarron C, Abdulkader I, Areses MC, GarciaPaz V, Leon L, Cameselle-Teijeiro J. Metastases of renal cell carcinoma to the thyroid gland with synchronous benign and malignant follicular cellderived neoplasms // Case Rep Oncol Med, 2013; 2013:485025

\section{Address:}

Arturs Ozolins

Pauls Stradins Clinical University Hospital, Department of General Surgery,

Pilsonu Street 13, LV-1002,

Riga, Latvia

E-mail: arturs.ozolins@me.com 

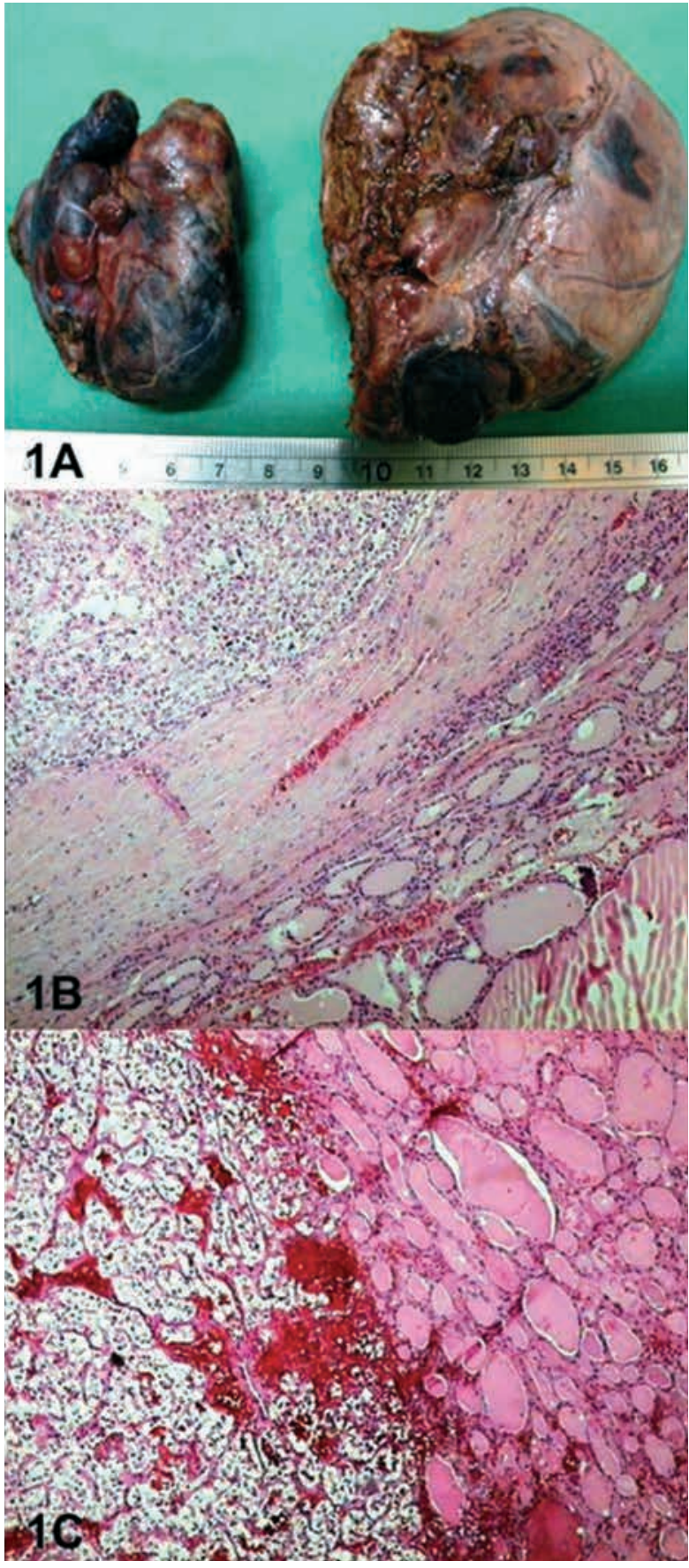

Fig. 1. Metastasis of renal clear cell carcinoma in thyroid gland. 1A, Gross view. Note the asymmetric nodular enlargement of left lobe. 1B, Overview of tissue structure. Haematoxylineosin (HE), original magnification (OM) 50x. 1C, Haemorrhages in metastatic tumour. Note also the rich vascularity. $\mathrm{HE}, \mathrm{OM} 100 \mathrm{x}$

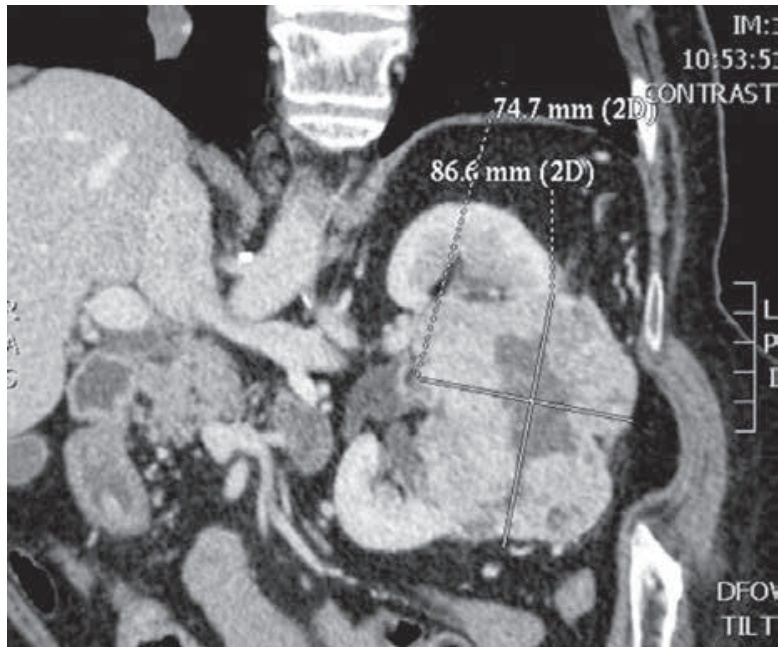

Fig. 2. Tumour mass in the posterior part of the left kidney by abdominal computed tomography scan

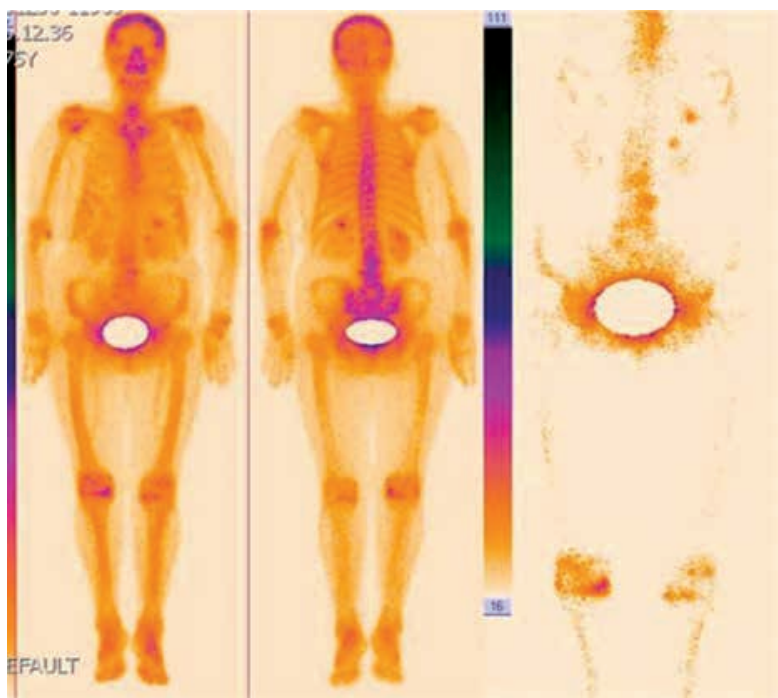

Fig. 3. Bone scintiscan without any evidence of skeletal metastasis 